\title{
The Accidental Checkmate: Understanding the Intent behind sharing Misinformation on Social Media
}

\author{
Ajay Agarwal \\ Department of Computer Science \& Engineering \\ DIT University, Dehradun \\ Mussoorie Road, Makka Wala \\ Dehradun - 248009 \\ Uttarakhand \\ India
}

\begin{abstract}
The bloom of COVID19 has resulted in the explosion of ripple pollens which have severely affected the world community in the terms of their multi-axial impact. These pollens, despite being indistinguishable, have a varied set of characteristics in terms of their origin and contribution towards the overall declining homeostasis of human beings. The most prominent of these pollens are misinformation. Various studies have been conducted, performed, and stochastically replicated to build ML-based models to accurately detect misinformation and its variates on the common modalities of spread. However, the recent independent analysis conducted on the prior studies reveals how the current fact-checking systems fail and fall flat in fulfilling any practical demands that the misinfodemic of COVID19 brought for us. While the scientific community broadly accepts the pandemic-like resemblance of the rampant misinformation spread, we must also make sure that our response to the same is multi-faceted, interdisciplinary, and doesn't stand restricted. As crucial it is to chart the features of misinformation spread, it is also important to understand why it spreads in the first place? Our paper deals with the latter question through a gametheory based approach. We implement a game with two social media users or players who aim at increasing their outreach on their social media handles whilst spreading misinformation knowingly. We take five independent parameters from 100 Twitter handles which have shared misinformation during the period of COVID19. Twitter was chosen as it is a prominent social media platform accredited to the major modality for misinformation spread. The outreach increment on the user's Twitter handles was measured using various features provided by Twitter - number of comments, number of retweets, and number of likes. Later, using a computational neuroscientific approach, we map each of these features with the type of neural system they trigger in a person's brain. This helps in understanding how misinformation whilst being used as an intentional decoy to increase outreach on social media, also, affects the human social cognition system eliciting pseudo-responses that weren't intended otherwise leading to realizing possible neuroscientific correlation as to how spreading misinformation on social media intentionally/unintentionally becomes a strategic maneuver to increased reach and possibly a false sense of accomplishment.
\end{abstract}




\section{Introduction}

The constant action of pouring academic research with ML-based methodologies in the research vessel designed for curing misinformation in the 21 st century has resulted in the overflow of hindered insight. With greater focus being targeted towards one-upping the prior's statistical metric without any significant contribution to the field whatsoever has resulted in the rampant rise of misinformation as a pandemic that is yet to be solved.[30].

The recent rise of COVID19 has realized the tools of machine learning and artificial intelligence are the contents of Pandora's box - attention-seeking with little to none actual shine. This observation, despite having its own rampant and dangerous manifestations in the real-world as seen with the spikes of healthbased misinformation, also has been echoed in academic papers from across the globe. Machine Learning and NLP-based methodologies to detect, classify, annotate, and finally restrict the spread of misinformation and its variants in a real and stochastic world fail and fall flat on many grounds stylometric limitations, evidence-based overreliance, context-based overreliance, adversarial attacks and constantly change in form. While each of these issues has been thoroughly discussed in research articles alike, we provide a succinct and simplistic overview regarding these issues viewing them from a more native intelligence-based approach.

1. Stylometric Limitations - Most NLP models are trained to identify author-specific features that help to trace the author for a given text. However, misinformation spread on social media is free from rigid and defined stylometric guidelines and grammatical rules. [1].

2. Evidence-based Overreliance - The current state-of-the-art fact verification classifiers are trained on a dataset that provides evidence for each claim, both for training and testing. It means that the NLP-models are asked to classify whether a statement is true or false, based on evidence providing the true statement behind the claim. This idiosyncrasy leads to no practically-usable result as misinformation spread on social media is not accompanied by its corresponding factual evidence.[2].

3. Adversarial Attacks - Automated fact-verification models are highly vulnerable to rule-based adversarial attacks that can fool the NLP model is changing its labels. These rule-based adversarial attacks are more common in the written vernacular and therefore are often found in misinformation posts on social media. [3].

4. Biased Datasets - The state-of-the-art datasets like FEVER and dEFEND etc. are mostly biased towards political events that were recorded in Western countries. More often than realized, these datasets contain their idiosyncratic methods of data annotation which make them less realistic for real-world environments.[4-6].

5. Modality Freeze - There doesn't exist any viable dataset or recorded anthology that has documented misinformation from non-text based modality. Misinformation is not frozen in the text-space. Recently countries like Singapore have witnessed the rise of "meme factories" that utilize this hybrid modality for spreading misinformation. Memes are 
characterized as visual components with context-based cues represented in the art style along with almost little to none textual content. [7-8].

This summarizes our short exploration in understanding the fallacies of the current ML-models in tackling the problem of misinformation. This also depicts how the unchecked over-dependence on any single field for tackling a global problem is not just a depicting of flawed thinking, but also defines our easily we, humans, end up trusting any new piece of observation (just like misinformation).

Accordingly, a justified question is raised - Which approach is best suited to tackle misinformation? No one single-approach should be pointed out in response to this question. A better phrasing of this question would be - What can we do as academic researchers to tackle misinformation? From our above analysis, it is clear that there exist two obstacles in our current path of research -

1. Multimodal, extensive, and context-aware mining and annotation of misinformation spread on social media platforms

2. Introduction of Inter-disciplinary methodological frameworks to identify possible reasons why misinformation is spread in one first place and what factors might motivate a person to share it?

Any future research in the field of misinformation must address the above two obstacles in their work and aim at eradicating them for root. As preached, this paper aims to address these obstacles, understand their impact, and provide valuable insight into the context of India. This country-specific context becomes extremely crucial for following the lean towards cultural neuroscience for the past few years.

\section{Proposed Methodology}

As stated prior, it is important for any new research aimed at tackling the problem of misinformation to address the above-mentioned questions in their research methodology. Therefore, our research shall aim to do the same as well.

When tackling a problem having resemblance with pandemic-like characteristics, it becomes prudent to take notes from history (or in this case our present). Misinformation is like a pandemic belonging to the likes of COVID19 and Ebola. Hence, it must be treated similarly. The response of the world's scientific community towards any pandemic that has occurred in history can be generalized in a two-step analogy document every change in the virus, and curb the spread-contain the infected. This analogy can, therefore, be extended towards our consensual response towards misinformation. In an attempt to do the same, our methodology is also divided into three parts - annotation, analysis, and mapping.

Annotation - In the first part of our methodology, we tackle the problem of 'modality freeze' in the case of misinformation. Just like COVID19, when viewed over a time-scale, we can see that 
stylometry and diction of misinformation have evolved and changed drastically over time. Not only that but in striking resemblance to COVID19, the list of possible mediums of spread and modality of communication for misinformation have also increased. The stakes are now running from social media to prime time news channels. Modality has evolved from simple textual cues to context-aware memes. Hence, in tracking the changes for the same, we present the MisMemoir dataset - which essentially tracks all the pieces of misinformation that were spread from March to September on Twitter India.

Analysis - The second crucial factor to weigh in in our research is "curbing the spread by containing the infected". In the context of misinformation, this statement loosely translates to - "the act of spreading misinformation is more dangerous than just believing in it and doing nothing". Given the increased vulnerability of average human beings to conforming with any piece of misinformation, it is likely that even before a cure is found, the curve is rounded i.e., people who even believe in any piece of misinformation should almost be refrained from sharing it or forwarding it ahead. Therefore, the question arises - what factors influence a user in sharing a piece of misinformation on social media (or in general any information).

Instead of looking at the direct approach of identifying key factors from a mix, we shall take the indirect route asking - what are the possible outcomes of sharing a piece of information on social media for a user? Social media platforms are based on the attention-net mechanisms i.e., any piece of information that can catch the attention of many is rewarded by an increase in the social metrics like followers, retweets, likes, and comments., whereas any piece of information that fails to garner the attention of many on the content is punished with a decrement in metrics. Hence, out of these two scenarios, any user when provided with a piece of information (even if it is misinformation) is highly likely to analyze the information's potential to garner vast attention, as per the former design.

In consequence of this observation, the following question can be posed for addressing in the second part of our methodology- Does share misinformation (intentionally/unintentionally) on social media act as a strategic move to increase one's social media ranking in place of providing increased attention currency to the platform? Also, how does this action rank against other actions involving sharing factual information on social media? Hence, for our purpose, we use a game-theory based approach and design a game in which sharing misinformation and sharing factual information becomes actions that can be taken in the game environment to maximize the user's metric rewards - followers, retweets, likes, etc. Such game-theoretic approaches have proved their merit in academic literature to analyze community online behaviors on social media platforms. [11-15].

Mapping - This finally brings us to the last segment of our methodology. Based on the existing academic literature on identifying neural circuits and cognitive feedbacks loops that are activated in context to any reward-specific action on social media platforms, we map the desired goals for the platform Twitter (gaining more retweets, likes, and followers) and the efficacy of sharing misinformation in achieving those goals, with the neural circuits in brains that would be activated if a particular goal is achieved.[16]. 
This summarizes the research methodology that applies to this current research. It is to be noted that Twitter has been chosen as a platform for study due to the lower number of possible metric rewards (only 3 - Followers, Likes, and Retweets) which allows accommodation of feasibility in conduction of this extensive methodology. A brief visual summarization of the research methodology has been shown in Figure 1.

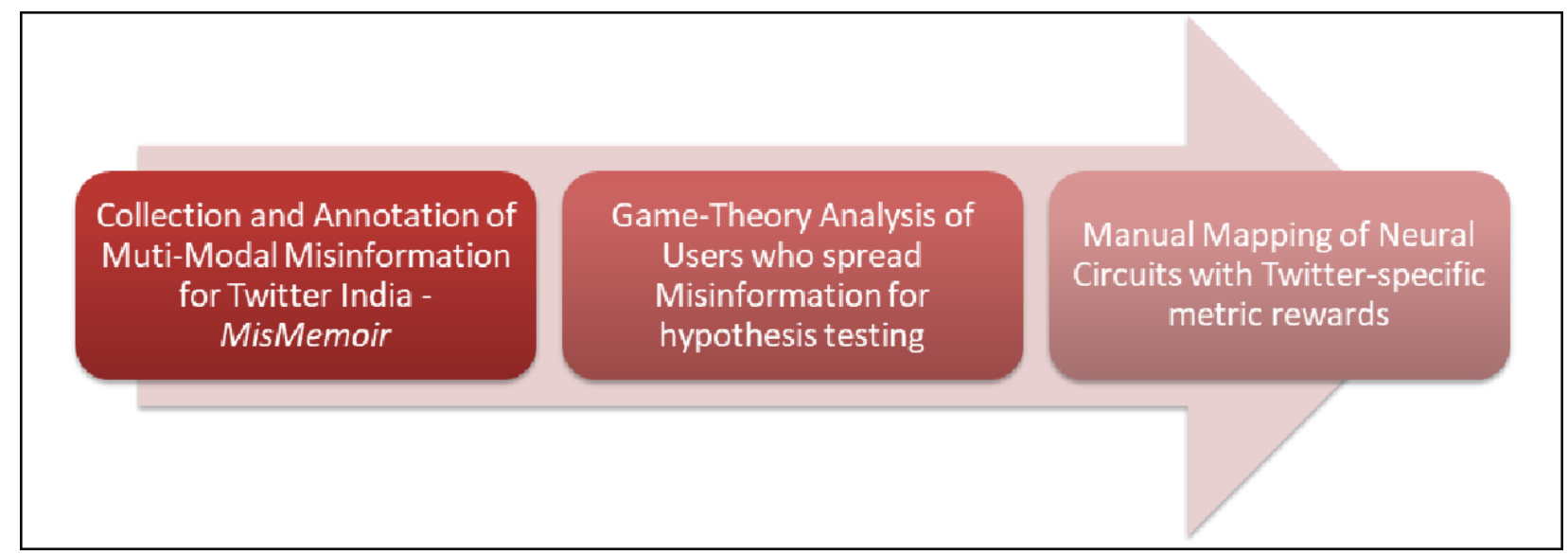

Fig 1. The above process diagram aptly summarizes the methodological plan opted for this research study.

\section{MisMemoir for Twitter India}

Since the first spotting of the misinformation pandemic during COVID19, the Indian Government assigned the job of fact-checking and misinformation detection on social media platforms to PIB (Public Information Bureau). As a result, dedicated social media handles were created in early March 2020 on all major platforms like Twitter, Facebook, Instagram, etc. under the aegis of PIBFactChecks to - "identify prevalent misinformation among public and fact-check the same by providing missing context, and/or required scientific evidence". The social media handles of PIBFactChecks soon became the face of India's campaign against the rising misinformation in the world. For our study, we focus our attention on the daily tweets posted out by @PIBFactCheck handle on Twitter which currently stands as the only government recognized anthology of all the misinformed claims that seen a significant rise during COVID19 and posed an imminent danger to public health and safety if a belief was associated with such claims.

MisMemoir India utilizes the tweets by PIBFactChecks as its first baseline. With the help of the Python Library, Twint, [9] tweets along with their corresponding meta-data like timestamp, likes count, retweet count, etc were collected for the Twitter handle - PIBFactChecks for the period of March to September 2020. These tweets provided the "fact labels" for the pieces of misinformation that are to be collected next. After the collection of the facts, the steps mentioned in Figure 2 were followed. 


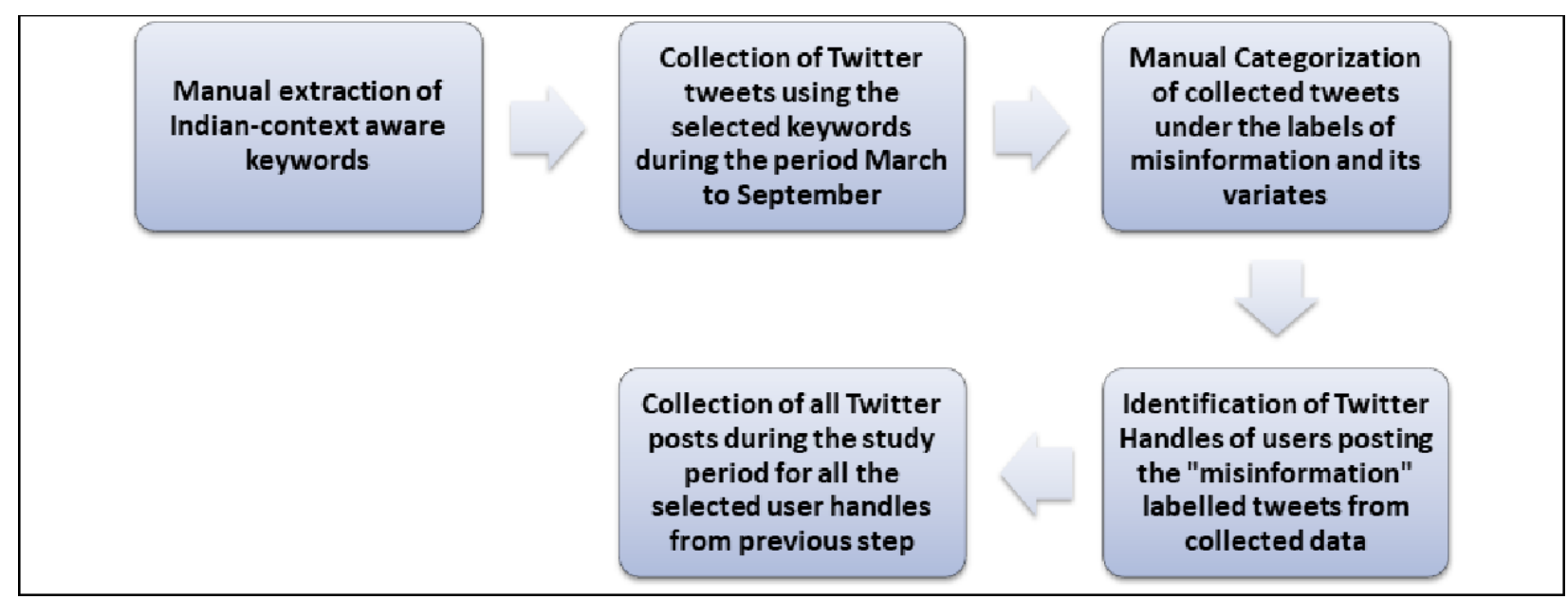

Fig 2. The above process diagram aptly visualizes the design flow and instruction that shall be followed in the collection of data and designing the MisMemoir dataset for Twitter India.

Manual extraction of keywords was required to keep in consideration the colloquial Indian context. The objective of capturing the characteristic Indian context is something that the latest and SOTA NLP models fail to capture. Therefore, manual intervention became a necessity. These keywords are most likely to be used in their original form by the Indian users in their course of discussion regarding the factual claim (in either informed or misinformed version), therefore, become the query searches for extracting tweets from the period of March to September that were posted on Twitter India. This dataset of more than 5000 tweets, then, were labeled under the categories of "misinformation" and "information" based on a doubleblind peer annotation process. Again, NLP technologies were not utilized due to the reason already mentioned in Section 1. As each collected tweet was followed by their corresponding metadata, the Twitter handles of the users spreading misinformation were identified which later was collected together for each corresponding factual claim. Lastly, utilizing the capacity of the Twint library, the Twitter tweets were extracted along with their corresponding metadata for each of the users (Twitter handle) responsible for spreading misinformation, utilizing the manually annotated keywords during the study period from March to September. These tweets were later utilized for our game-theory study.

This summarizes the extraction, annotation, and collection process of the MisMemoir India (Twitter version) dataset. As evident as it may be, the design of this dataset is intentionally complex within limits. The main aim behind the inherent complexity is to capture all prominent features of a misinformed claim along with the characteristics of the person responsible for spreading it. A simplified version explaining the navigation process through MisMemoir is shown in Figure 3. 


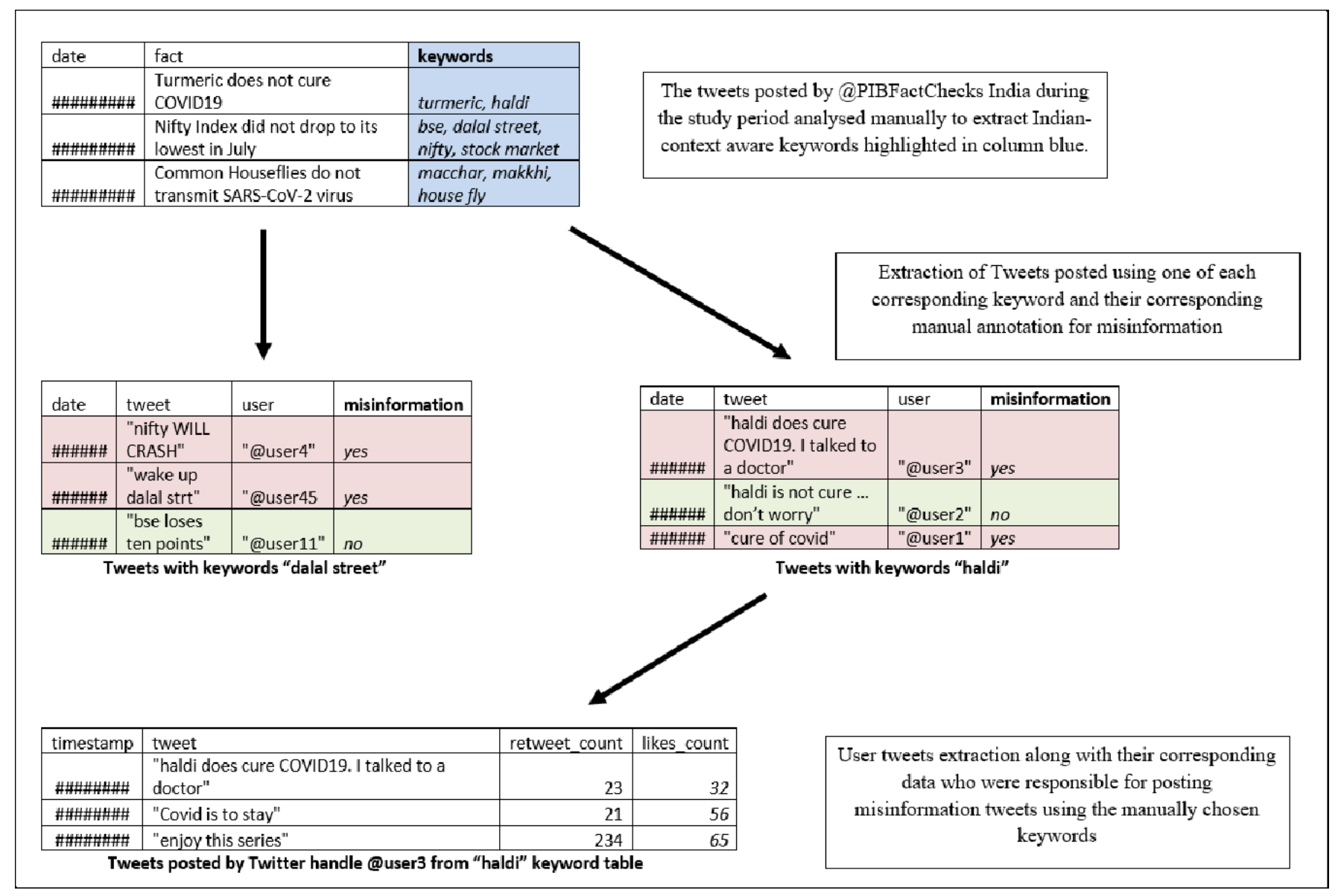

Fig 3. The above figure provides a visual understanding of how different tables in the MisMemoir Twitter India dataset are related to each other. Notice how this tabular relationship follows the design principles highlighted in Figure 2.

With MisMemoir created, we shall now move to the second wing of our research - analysis using a gametheory based approach.

\section{Game-Theoretic Analysis of MisMemoir Users}

The Indian Nodal Agency, PIB, established its Twitter handle @ PIBFactCheck on December 19th, 2019. Since then, the handle has posted around 528 tweets till the end of September 2020. Each of these tweets was targeted on fact-checking one or the other misinformation claim that had been circulating rampantly around the localized period. After the process of MisMemoir formation, it was realized that around 284 Twitter handles were responsible for spreading misinformation on an average for each of the fact-checked claims. Hence, the obvious question 
that arose was - Why did these Twitter handles spread misinformed claims? It is important to realize that whilst our focus is not to concentrate on the intention behind these posts, but rather understand the factors that could unintentionally influence a person to share such posts. In the context of social media, the factor that we aim to study is attention, measured by the metrics of follower count, and likes count. Hence, the hypothesis that we seek to validate is -

"Does the action of sharing misinformation on social media divert more than usual attention towards the user than before? Also, is this increased attention quantifiable in the platform's user metrics?"

For the same, we design a simple game for two social media users - one user from the MisMemoir dataset, and the other anonymous user. This anonymous user was a randomly selected Twitter handle that fulfilled the following criteria -

1. Belonged to India

2. Did not belong to the MisMemoir dataset

3. Partially or Wholly resembled the user metrics as that of its pair

4. On manual verification, was not involved in spreading misinformation during the COVID19 period

Both of these users were players of a game where their ultimate goal was to increase their organic outreach to the maximum levels through two actions - posting misinformation-laden posts and posting objective posts (any post that is not-laden with misinformation). The number of variables was optimized to account for the incorporation of game theory in the research problem. The number of variables we included was- likes count on second-to-most-recent post $l$, followers count $f$, posts count $n$, comments count on second-to-most-recent post $c$, and sentiment in comments (in decimal value) denoted as $s$. The positive sentiment in the misinformation-laden posts reflects confirmation with the misinformation claim, whereas negative sentiment in the misinformation-laden posts reflects dissent with the misinformation claim.

To denote the players in our game along with their corresponding set of actions (two) that they can perform, we use the notation of game action models denoted by $p 1$ and $p 2$, where

$$
\begin{aligned}
& p_{1} \in U \\
& p_{2} \in W
\end{aligned}
$$

In the aspect of game-theory, cost function $L$ becomes the measure of misinformation spread in the community as represented in Equation 1 and the payoff function, denoted by $J$, is the social outreach of the user as represented in Equation 2. The optimum community will be the user which harnesses the optimum social outreach with the least count of misinformation-laden posts. 


$$
L=\frac{l f+c s}{\frac{n}{2}(s+1)}\left(\frac{n l s c}{f}\right)
$$

This forms the mathematical basis of our hypothesis as shown below. The action chosen by our user affects the corresponding payoff and cost function by an arbitrary increase/decrease.

H0- Misinformation attracts social attention, hence the payoff function and cost function increases by an arbitrary value if action 1 is performed ( posting misinformation) and decreases if action 2 is performed (posting objective content).

H1- Misinformation does not attract social attention, hence the payoff function and cost function decreases by an arbitrary value if action 1 is performed (posting misinformation) and increases if action 2 is performed (posting objective content).

Note, that in our alternate hypothesis, we stated the decrement in the cost function if action 1 is performed as the cost becomes a consequence of the decreased payoff from the resulting action - fewer people are attracted by the misinformation posts, and hence misinformation fails to spread to a larger mass.

Being a two-person cooperative game, the move of each player is dependent on the previous move and its result on the other player.[10]. This is denoted as follows for each player $p 1$ and $p 2$ as following -

$$
\begin{aligned}
& p_{1}=\dot{U}\left(p_{2}\right) \\
& p_{2}=\dot{U}\left(p_{1}\right)
\end{aligned}
$$

At last, our payoff function $K$ can now be defined for both our null and alternate hypothesis as follows as Equation 2(a) and Equation 2(b) as follows -

$$
\begin{aligned}
& J\left(y, \dot{U}\left(p_{2}\right), p_{2}\right) \geq J\left(p_{1}, p_{2}\right) \forall p_{1} \in U, p_{2} \in W \\
& J\left(y, \dot{U}\left(p_{2}\right), p_{2}\right) \leq J\left(p_{1}, p_{2}\right) \forall p_{1} \in U, p_{2} \in W
\end{aligned}
$$

Let's assume that $y(t)$ denotes the total number of followers for both the players at a given point $t$ in the move-set, then -

$$
y(t+1)=J\left(y(t), p_{1}(t), p_{2}(t)\right)
$$


This new function denoted by $J$ can essentially be re-written in terms of the payoff function $K$ shown in Equation 2 and cost function $L$ as shown in Equation 1.

$$
J=K\left(y(t), p_{1}(t), p_{2}(t)\right)-L\left(y(t), p_{1}(t), p_{2}(t)\right)
$$

\section{Results}

Based on the game-theoretic model build based on our null and alternate hypothesis, the final task to complete the second-segment of our methodology to chart out the averaged parameter values for each of the 284 misinformation spreader Twitter handles and their anonymous pairs.

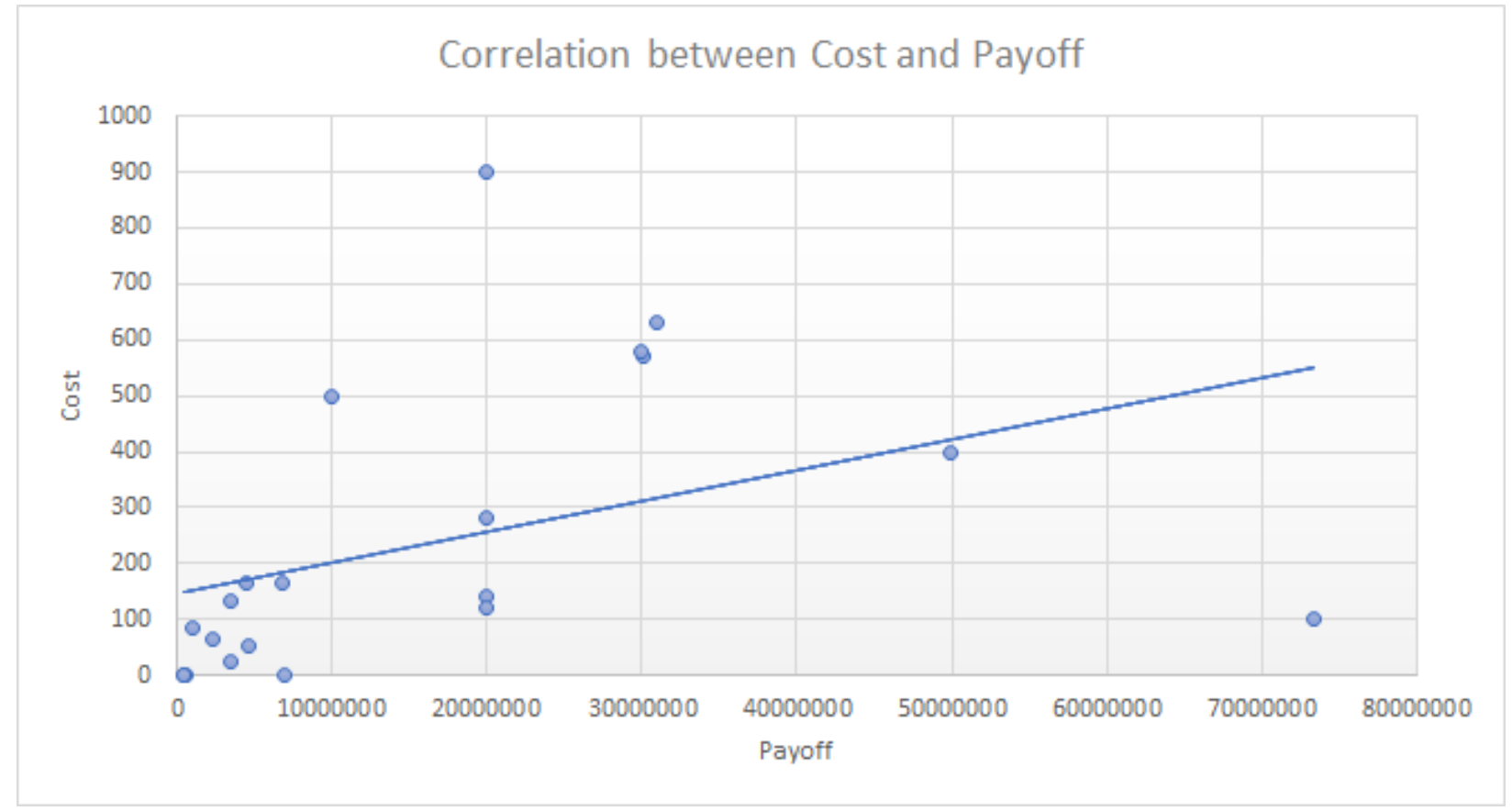

Fig 4. The above graph depicts the correlation between the cost and the payoff function for the null hypothesis, hence discarding the alternate hypothesis. The correlation coefficient obtained between the cost and payoff function for the null hypothesis is $\mathbf{0 . 3 9 9 5 9}$, while that for the alternate hypothesis payoff function is negative $\mathbf{0 . 1 3 2 3}$

As visible from Figure 4, there exists a positive linear correlation $(=0.39959)$ between the cost and payoff function, which proved our null hypothesis. This signifies that misinformation does attract the attention of the social media users, and consequently increases the social media outreach of the person spreading misinformation 


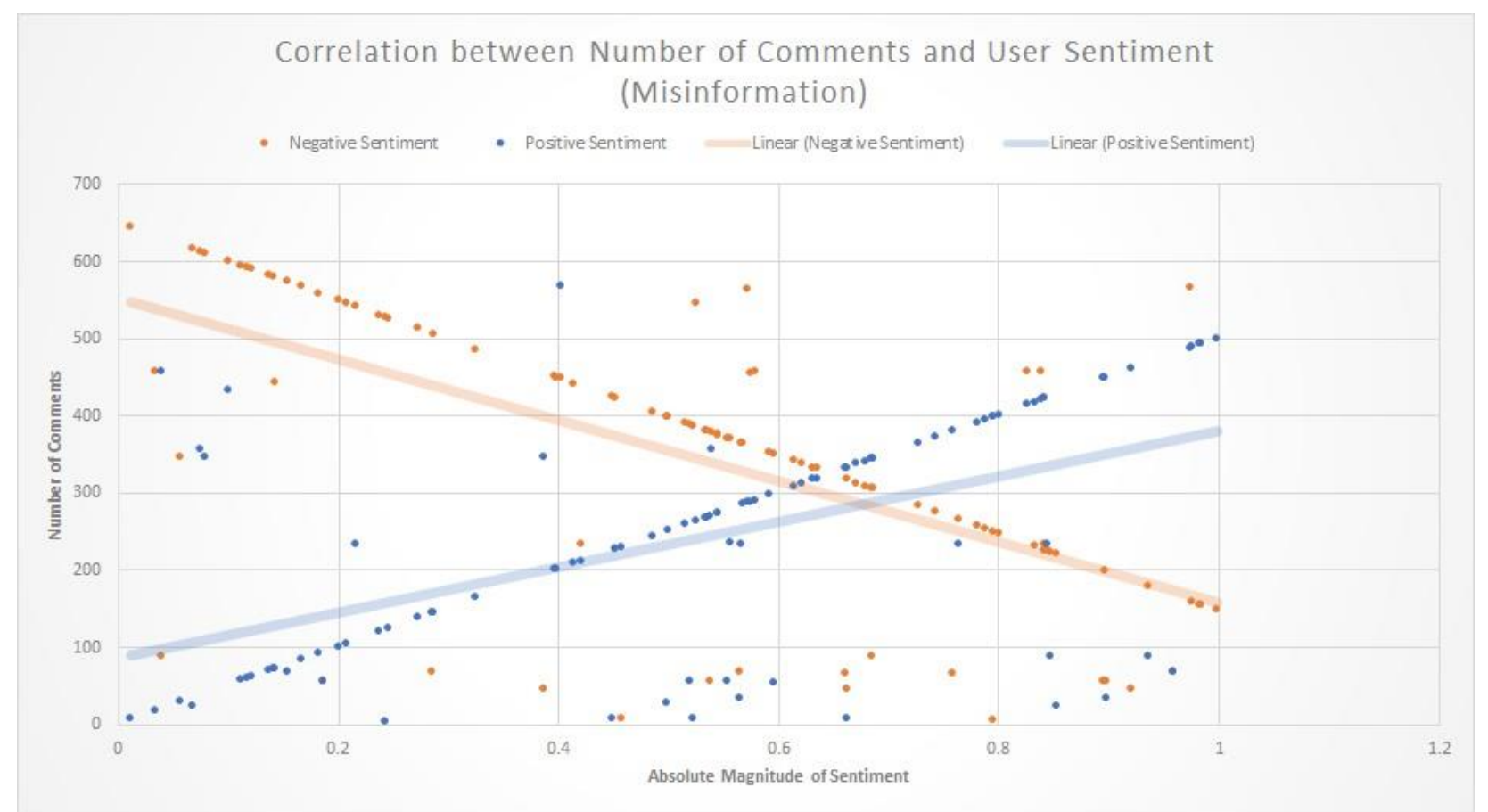

Fig 5. Notice how the increase in the number of comments on player 1 posts correlates with an increase in the positive sentiment of the user and decrease in negative sentiment which affirms the claim that comments on posts laden with misinformation carry an increasing positive sentiment reflecting peoples' confirmation in misinformed statements. 


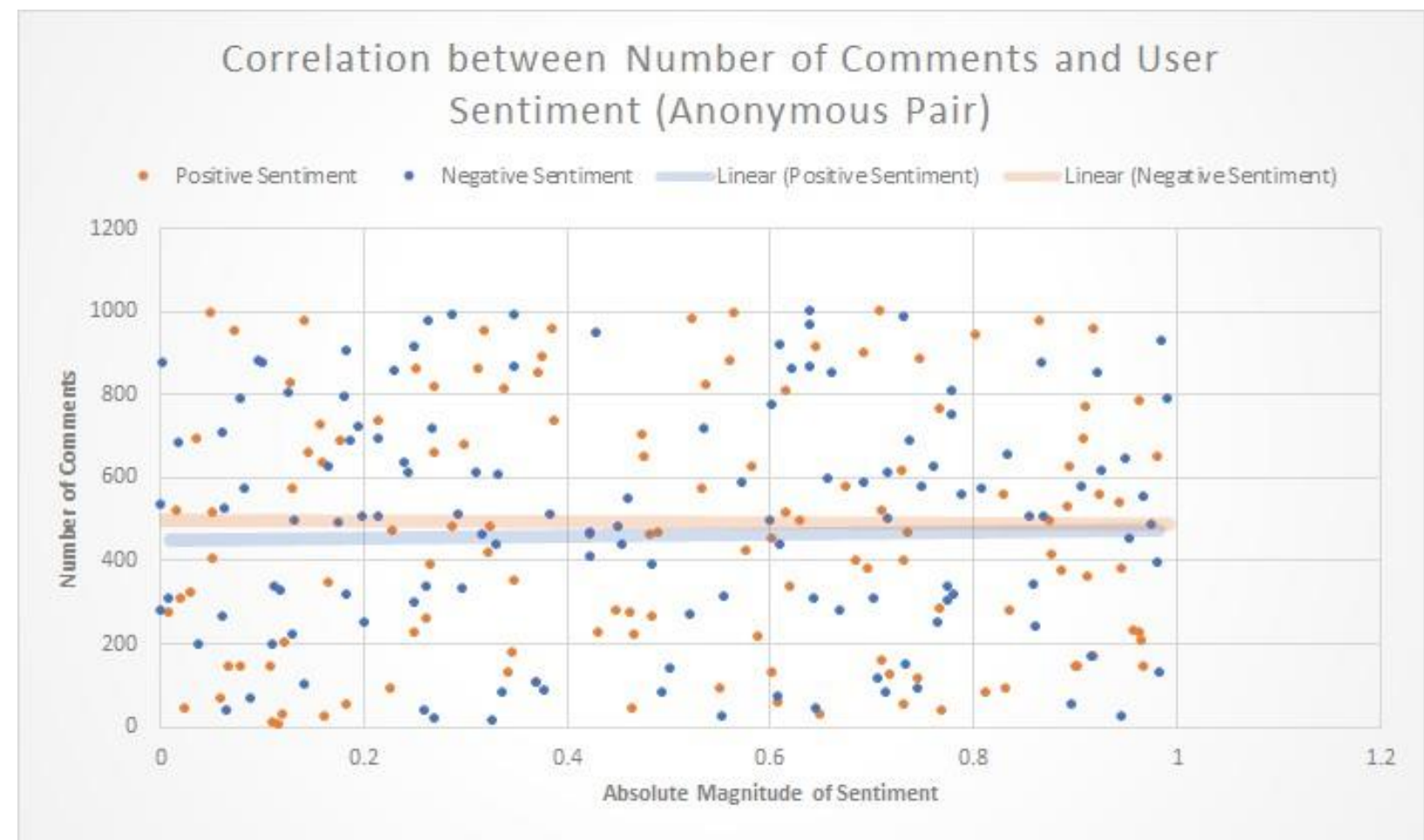

Fig 6. Notice the presence of almost no significant correlation between the number of comments and user sentiment for player 2. This shows that a strategy based on increasing social outreach against a player using the action of posting misinformation-laden posts is almost useless especially when the payoff is based on the proven null hypothesis.

\section{Neuroscientific Inferences}

Social media platforms like Twitter and Facebook are notoriously famous for their social outreach metrics which is a quantitative measure of their platform-specific reward cues for gathering focused attention of users on the platform and increasing their stickiness with the product. These reward cues are minimalist and are often criticized for their vagueness in defining "social outreach" and algorithm heavy dependence. Despite their constant rebranding in the tags of "retweets in Twitter", "likes in Facebook" or "shares in Instagram", these cues have succeeded consistently in being able to act as trigger points for eliciting a virtual response from the reward circuits in our brains - making us identify the need of such cues as a fundamentalist requirement of our body, and their acquirement as gratification and fulfillment of such. As a result, the task for identifying the cause of online behavior has to be integrated with understanding and identifying how the brain perceives the said behavior.[16]. 
Prior studies in the field of neuroscience have cemented their acceptance and understanding of social media reward cues that have a direct correlation with the response and activity levels witnessed in the reward-circuit regions of our brain. Established that the human reward system is easy to fool in identifying pseudo-social needs as fundamentalist requirements, we can safely say that any activity, action, or task that has the potential to fool the reward circuitry of the human brain in identifying the completion of the given action as a requirement for our body raises a red-flag for the virtues and merits the given activity. This statement becomes increasingly jarring when we introspect the cases of player 1 in our everyday life - people who spread misinformation.

Reiterating the theme of the paper, whilst player 1 harnessed more social outreach by spreading misinformation on social media and wins the defined game every-time, from the neuroscientific point-ofview, he is accidentally drawn into a checkmate by the set of strategies he uses - as his brain with time identifies the need for harnessing social outreach as fundamental requirements and assigns credibility to the strategy of sharing misinformation in fulfilling the given requirement. This not only damages his analytical sense and weakens his System1-System2 thinking as defined by Kahneman [17-19], but also increases the susceptibility of being addicted to this activity as his brain's activity resembles the same response triggered by a person administering the use of narcotics.[27].

The brain's reward loop largely resides in three significant regions - ventral striatum (VS), ventral tegmental area (VTA), and ventromedial prefrontal cortex (vmPFS). [25-26]. While VS and VTA are well-defined anatomically, vmPFS is a largely ill-defined region having a broad general disparity for its location and dimension.

Let's understand briefly how player 1 draws himself into an "accidental checkmate" whilst continuing to believe that he won the game. When player 1 broadcasts any content (in this case misinformation) on social media, the VTA region is activated as shown in Figure 7. Due to existing cognitive biases [28] that reside in our brain, the post laden with misinformation issued by player 1 harnesses more social attention than that by player 2 . Throughout gradient time, the "reward cues" on the social media platform climb in trend. The number of likes, followers, and positive sentiment in the comments increases - which significantly induces high activity in the region of vmPFS as shown in Figure 8. The vmPFS region identifies this uptrend as receiving positive social feedback [23] and the general agreement [24] of the society with the views of player 1 . When player 1 contrasts his progress against that with player 2 in "the social outreach game", his higher outreach numbers (likes count, positive sentiment in comments, followers count) elicits a positive response from the VS region of the brain as shown in Figure 9 - which identifies it as a "win" in a perceived "competition" or "comparison" of success [20-22]. Every time player 1 makes an act of spreading misinformation, the above neural loop is triggered, and with each trigger, a flood of dopamine is released in the body - creating a constant sense of euphoric high and a false sense of achievement. The position of providing a neurochemical commentary on this theoretic game to understand the role of dopamine lies outside this paper. Dauntingly enough, it is well-known, both in academia and society, how the need for this euphoric "dopamine" is fulfilled using false substances and wrongful activities effective enough in creating a fog of pseudo-achievement and shaky success. Unfortunately, in this case, player 
1 intentionally/unintentionally established a new action for acquiring the sweet taste of dopamine sharing misinformation on social media.

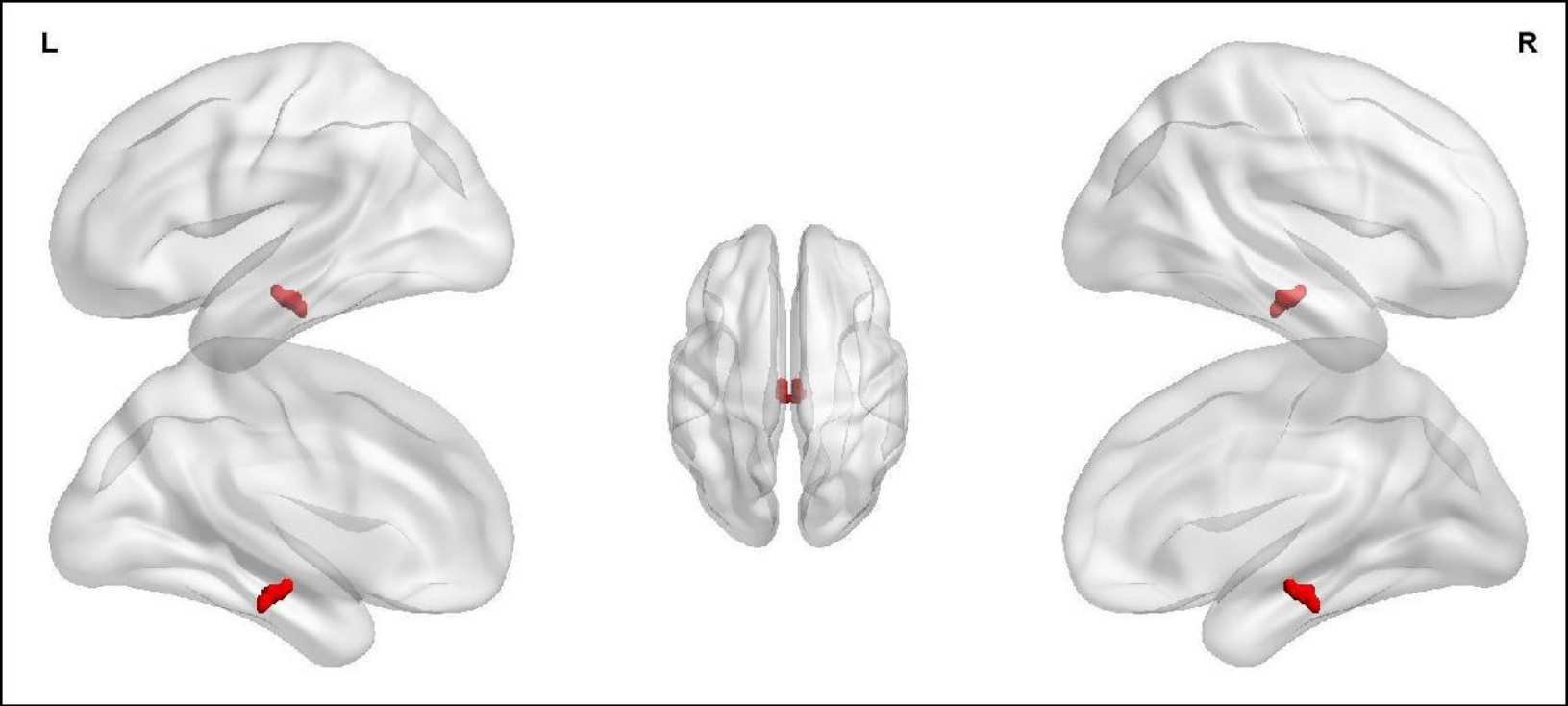

Fig 7. Using the MATLAB BrainNet [29], the region of the Ventral Tegmental Area (VTA) has been highlighted. Ventral Tegmental Area registers high-dopamine-inducing activity when the user broadcasts any information on social media that is successful in harnessing mass user appeal and affirmation.

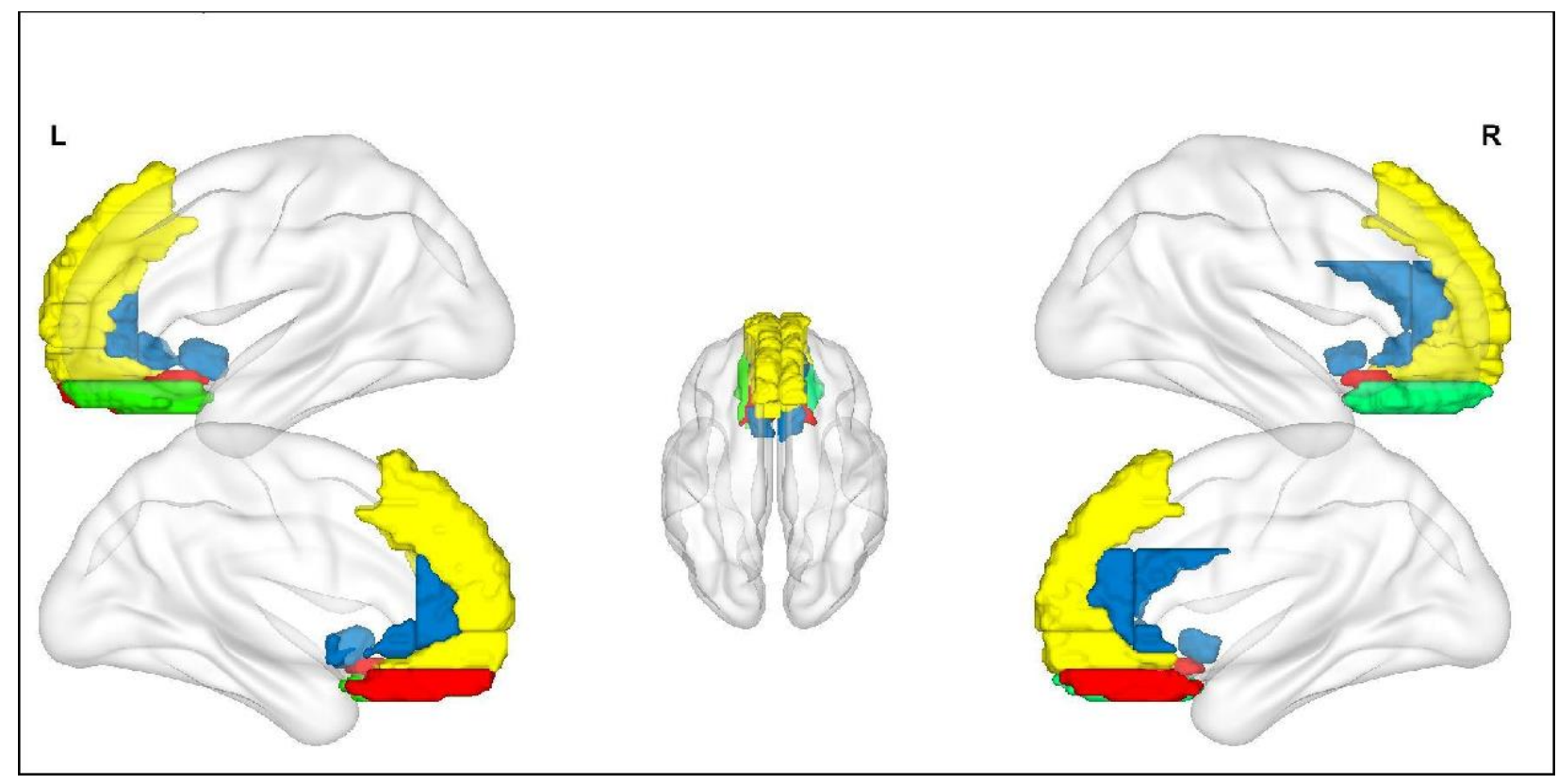

Fig 8. Using the BrainNet MATLAB [29], the generally accepted definition of Ventromedial Prefrontal Cortex (vmPFS) is highlighted. vmPFS registered high-dopamine-inducing 


\section{activity when player 1 observes his increasing "social outreach" as reflected in the upward trend of his "reward cues like followers and likes count" as a result of his action of spreading misinformation.}

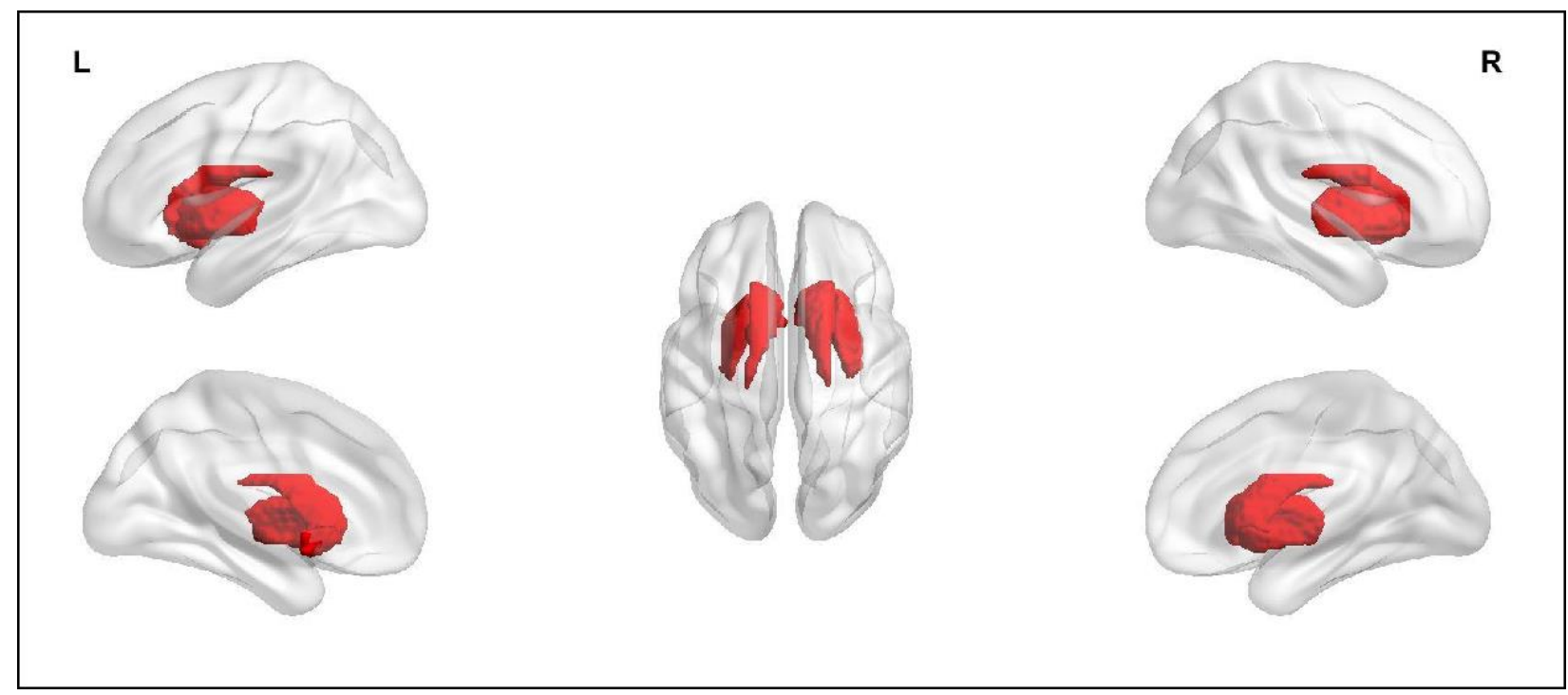

Fig 9. Using MATLAB BrainNet [29], the region of Ventral Striatum (VS) has been highlighted. The VS region registers high-dopamine-inducing activity when player 1 contrasts his successful progress over player 2 in the "social outreach game".

\section{Conclusion}

The misguided association of success and reward with activities with diminished stakes and virtues has been common-ground in our society. However, most of these activities have been independent of the network of the user and only bore physical manifestations on the user of such activities. In contrast to the common baseline, our paper establishes a new action - "sharing misinformation on social media" as a neuroscientific question. The paper successfully refreshes the general understanding of a "pandemic" and how the continued misinterpretation of the same for "misinformation" has rendered years of academic research in a practical limbo. Following a rather critique view is the comprehensive methodology of this paper which is interdisciplinary, proves its merit in diving into the psychological catacombs of a person that shares misinformation on social media establishing the remotest possibility of addiction to the same.

While the paper presents a discourse on the psyche behind a person sharing misinformation on social media intentionally or unintentionally, the future studies in these caves must be based on choosing specific and nuanced routes to understanding even the most fundamental questions like - Does the action of sharing misinformation intentionally differs in neuroscientific activity than those who do unintentionally? Does lexicography reflect intent-based variations when sharing misinformation? How does cognitive activity vary for people sharing misinformation online than to those who share offline? How are social rewards perceived when messaging 
services act as the modality of spread? How does cognitive activity vary when receiving misinformation through broadcasting services with a pre-established sense of credibility and merit?

The questions to discuss and research extend beyond our limits till we associate the right methodological practices and promote interdisciplinary approaches for problems persistent and complex. Misinformation is a "controllable pandemic" till the time our academic conscience attacks the problem whilst increasing the research volume gradient justifiably. Any digression from this path will only result in one outcome misinformation being written as a bold foreword in our "new normal of 21 st century".

\section{References}

1. Schuster, Tal, et al. "The Limitations of Stylometry for Detecting Machine-Generated Fake News." Computational Linguistics (2020): 1-12.

2. Purian, Ronit, Shuyuan Mary Ho, and Dov Te'Eni. "Resilience of Society to Recognize Disinformation: Human and/or Machine Intelligence." (2020).

3. Thorne, James, et al. "Evaluating adversarial attacks against multiple fact verification systems." Association for Computational Linguistics, 2020.

4. Schuster, Tal, et al. "Towards debiasing fact verification models." arXiv preprint arXiv:1908.05267 (2019).

5. Schuster, Tal, et al. "Are we safe yet? the limitations of distributional features for fake news detection." arXiv preprint arXiv:1908.09805 (2019).

6. Schiller, Benjamin, Johannes Daxenberger, and Iryna Gurevych. "Stance Detection Benchmark: How Robust Is Your Stance Detection?." arXiv preprint arXiv:2001.01565 (2020).

7. Shin, Jieun, et al. "The diffusion of misinformation on social media: Temporal pattern, message, and source." Computers in Human Behavior 83 (2018): 278-287.

8. Brennen, J. Scott, et al. "Types, sources, and claims of COVID-19 misinformation." Reuters Institute 7 (2020): 3-1.

9. Zacharias, C., and F. Poldi. "GitHub-twintproject/twint: an advanced Twitter scraping \& OSINT tool written in Python that doesn't use Twitter's API, allowing you to scrape a user's followers, following, tweets and more while evading most API limitations, February 2020." (2020).

10. Nash, John. "Two-person cooperative games." Econometrica: Journal of the Econometric Society (1953): 128-140.

11. Hajibagheri, Alireza, et al. "Community detection in social networks using information diffusion." 2012 IEEE/ACM International Conference on Advances in Social Networks Analysis and Mining. IEEE, 2012.

12. Zhan, Felix, et al. "Incorporating Game Theory and Nash Equilibrium Concepts to Predict Social Network Community Behaviors." University of Nevada Las Vegas AEOP/STEM/REAP/RET Programs Technical Report 2 (2017): 26-30. 
13. Fortetsanakis, Georgios, Ioannis Dimitriou, and Maria Papadopouli. "A game-theoretical analysis of wireless markets using network aggregation." IEEE Transactions on Mobile Computing 16.3 (2016): 602-616.

14. Xia, Feng, et al. "A signaling game for uncertain data delivery in selfish mobile social networks." IEEE Transactions on Computational Social Systems 3.2 (2016): 100-112.

15. Zhan, Felix, et al. "Prediction of online social networks users' behaviors with a game theoretic approach." 2018 15th IEEE Annual Consumer Communications \& Networking Conference (CCNC). IEEE, 2018.

16. Meshi, Dar, Diana I. Tamir, and Hauke R. Heekeren. "The emerging neuroscience of social media." Trends in cognitive sciences 19.12 (2015): 771-782.

17. Tversky, Amos, and Daniel Kahneman. "Judgment under uncertainty: Heuristics and biases." science 185.4157 (1974): 1124-1131.

18. Kahneman, Daniel. Thinking, fast and slow. Macmillan, 2011.

19. Tversky, Amos, and Daniel Kahneman. "The framing of decisions and the psychology of choice." science 211.4481 (1981): 453-458.

20. Fliessbach, Klaus, et al. "Social comparison affects reward-related brain activity in the human ventral striatum." science 318.5854 (2007): 1305-1308.

21. Mataix-Cols, David, and Odile A. van den Heuvel. "Common and distinct neural correlates of obsessive-compulsive and related disorders." Psychiatric Clinics 29.2 (2006): 391-410.

22. Strait, Caleb E., Brianna J. Sleezer, and Benjamin Y. Hayden. "Signatures of value comparison in ventral striatum neurons." PLoS Biol 13.6 (2015): e1002173.

23. Morelli, Sylvia A., Jared B. Torre, and Naomi I. Eisenberger. "The neural bases of feeling understood and not understood." Social Cognitive and Affective Neuroscience 9.12 (2014): 18901896.

24. Davey, Christopher G., et al. "Being liked activates primary reward and midline self-related brain regions." Human brain mapping 31.4 (2010): 660-668.

25. Fareri, Dominic S., and Mauricio R. Delgado. "Social rewards and social networks in the human brain." The Neuroscientist 20.4 (2014): 387-402.

26. Ruff, Christian C., and Ernst Fehr. "The neurobiology of rewards and values in social decision making." Nature Reviews Neuroscience 15.8 (2014):549-562.

27. Koob, George F., and Nora D. Volkow. "Neurocircuitry of addiction." Neuropsychopharmacology 35.1 (2010): 217-238.

28. Soon, Carol, and Shawn Goh. "Fake news, false information and more: Countering human biases." Institute of Policy Studies (IPS) Working Papers 31 (2018).

29. Xia, Mingrui, Jinhui Wang, and Yong He. "BrainNet Viewer: a network visualization tool for human brain connectomics." PloS one 8.7 (2013): e68910.

30. Roozenbeek, Jon, et al. "Susceptibility to misinformation about COVID-19 around the world." Royal Society Open Science 7.10 (2020): 201199. 